The Astrophysical Journal, 475 :L77-L80, 1997 February 1

(C) 1997. The American Astronomical Society. All rights reserved. Printed in U.S.A.

\title{
MILLIMETRIC GROUND-BASED OBSERVATIONS OF COSMIC MICROWAVE BACKGROUND ANISOTROPY
}

\author{
L. Piccirillo, ${ }^{1}$ B. Femenía, ${ }^{2}$ N. Kachwala, ${ }^{1}$ R. Rebolo, ${ }^{2}$ M. Limon, ${ }^{1}$ C. M. Gutiérrez, ${ }^{2}$ \\ J. Nicholas, ${ }^{1}$ R. K. Schaefer, ${ }^{1}$ AND R. A. WATSON ${ }^{2,3}$ \\ Received 1996 June 21; accepted 1996 November 14
}

\begin{abstract}
First results of a cosmic microwave background (CMB) anisotropy experiment conducted at the Observatorio del Teide (Tenerife, Spain) are presented. The instrument is a four channel (3.1, 2.1, 1.3, and $1.1 \mathrm{~mm}){ }^{3} \mathrm{He}$ bolometer system coupled to a $45 \mathrm{~cm}$ diameter telescope. The resultant configuration is sensitive to structures on angular scales $\sim 1^{\circ}-2^{\circ}$. We use the channels at the two highest frequencies for monitoring the atmosphere and apply a simple method to subtract this contribution in channels $1(3.1 \mathrm{~mm})$ and $2(2.1 \mathrm{~mm})$. The most intense structure at these two frequencies is the Galactic crossing, with peak amplitudes of $\sim 350 \mu \mathrm{K}$. These crossings have been clearly detected with the amplitude and shape predicted. This demonstrates that our multifrequency observations allow an effective assessment and subtraction of the atmospheric contribution. In the section of data at high Galactic latitude we obtain sensitivities $\sim 40 \mu \mathrm{K}_{\text {beam }}^{-1}$. The statistical analyses show the presence of common signals between channels 1 and 2 . Assuming a simple Gaussian autocorrelation model with a scale of coherence $\theta_{c}=1.32$ for the signal, a likelihood analysis of this section of data reveals the presence of fluctuations with intrinsic amplitude $C_{0}^{1 / 2}=76_{-32}^{+42} \mu \mathrm{K}$ (with a $68 \%$ confidence limit including an $\sim 20 \%$ calibration uncertainty). Since residual atmospheric noise might still contaminate our results, we also give our result as an upper limit of $118 \mu \mathrm{K}$ at a $95 \%$ confidence limit.
\end{abstract}

Subject heading: cosmic microwave background — cosmology: observations

\section{INTRODUCTION}

Measurements of fluctuations in the cosmic microwave background $(\mathrm{CMB})$ radiation provide one of the most direct tests of theories for the formation of structure in the universe. On large angular scales where the fluctuations are produced by the SachsWolfe effect in the last scattering surface, the overall normalization $Q_{\mathrm{rms}-\mathrm{Ps}} \sim 18 \mu \mathrm{K}$ is well established by the COBE DMR (Bennett et al. 1996) and the Tenerife beam-switching (Hancock et al. 1996) experiments. On smaller scales $\left(\sim 1^{\circ}\right)$ the acoustic effects on the last scattering surface are expected to enhance the level of fluctuations relative to those at larger angular scales, giving rise to a feature in the power spectrum of the CMB fluctuations, the so-called "Doppler peak," with a position and shape which depends on the values of cosmological parameters (the curvature of the universe, the Hubble constant, and the baryonic contribution). The current observations have recently started to allow a first determination of the position and height of this peak (Netterfield et al. 1996; Scott et al. 1996). Nevertheless more observations are needed to reduce uncertainties due to residual atmospheric signals or diffuse Galactic contamination. The main aim of the present experiment is to fill the gap in $\ell$ space between the Tenerife beam-switching experiments (Hancock et al. 1994) with a flat spectrum weighted $\ell=20 \pm 8$, and both the ACME South Pole (Gundersen et al. 1995) $\left(\ell=68_{-32}^{+38}\right)$ and the Saskatoon experiment (Netterfield et al. 1995). The experiment consists in a multichannel bolometer detector which is sensitive to multipoles in the range $\ell=38-77$ with the maximum sensitivity at $\ell=53$. Here we present results of our first observing campaign in the summer of 1994.

\footnotetext{
${ }^{1}$ Bartol Research Institute, University of Delaware, Newark, DE 19716.

${ }^{2}$ Instituto de Astrofísica de Canarias, 38200 La Laguna, Spain.

${ }^{3}$ University of Manchester, Nuffield Radio Astronomy Laboratories, Jodrell Bank, Macclesfield, Cheshire, SK11 9DL, UK.
}

\section{INSTRUMENTAL SET-UP}

The instrument is described in detail elsewhere (Piccirillo 1991; Piccirillo \& Calisse 1993). In summary, the optics consist of a primary off-axis parabolic mirror $(45 \mathrm{~cm}$ diameter $)$ coupled to a secondary off-axis hyperbolic mirror $(28 \mathrm{~cm}$ diameter $)$. The detector is a four-channel photometer equipped with ${ }^{3} \mathrm{He}$ bolometers working at $0.33 \mathrm{~K}$. The bands are centered at 3.3, 2.1, 1.3, and $1.1 \mathrm{~mm}$ wavelengths (channels $1,2,3$, and 4) as defined by a combination of resonant mesh filters. The instrumental noise is 3 , $1,1.6$, and $1.2 \mathrm{mK} \mathrm{s}^{1 / 2}$ in thermodynamic units for channels $1-4$, respectively. High-frequency leaks are blocked by a combination of fluorogold, black polyethylene, and Pyrex glass filters. Laboratory tests have been done to check for the absence of significant leaks in the filters. The telescope is surrounded by $45^{\circ}$ aluminum radiation shields fixed to the ground. The beam and sidelobes have been extensively checked by placing a distant Gunn source oscillating within channel 1 band. An accurate bidimensional map of the beam shape has been obtained $\left(20^{\circ} \times 20^{\circ}\right)$. We also studied the far-field sidelobe structures down to about $-72 \mathrm{~dB}$. These analyses show that the beam response can be approximated by a Gaussian with FWHM $=2.4$ and that no significant sidelobes are found.

The observing strategy consists of daily drift scans done at fixed position in azimuth and elevation. The beam throw in the sky is achieved by fixing the secondary mirror and chopping sinusoidally the primary mirror, according to $\theta(t)=\theta_{0}, \phi(t)=\phi_{0}+$ $\alpha_{0} \times \sin \left(2 \pi f_{w} t+\psi\right)$, where $\phi(t)$ and $\theta(t)$ denote the azimuth and elevation, respectively, $\left(\phi_{0}, \theta_{0}\right)=\left(0^{\circ}, 78.5\right)$ is the initial position of the antenna (see next section), $\alpha_{0}=2.6$ is the zero-to-peak azimuthal chopping amplitude at a reference frequency $f_{w}=4 \mathrm{~Hz}$. The demodulation of the signal is done online in software by evaluating the amplitude of the first $(4 \mathrm{~Hz})$ and second $(8 \mathrm{~Hz})$ harmonic of the reference frequency. The resulting sky pattern for the transit of a pointlike source resembles, respectively, the well-known two-beam and three-beam response. In this paper we only deal with the second-harmonic demodu- 
lated data. We measured a stable DC offset (about 10 and $15 \mathrm{mK}$ for $\mathrm{Ch} 1$ and $\mathrm{Ch} 2$, respectively) which is partly due to the arc-shaped motion of the beam in the sky, i.e., the axis of rotation of the wobbling mirror is not exactly vertical, so the center position of the beam is a few arcminutes higher than the two lateral position. The absolute pointing error has been estimated by observing the millimeter emission of the full Moon; conservatively we assumed that this error was not larger than the diameter of the moon $\left(\sim 30^{\prime}\right)$. The calibration constants, for each channel, have been chosen to give $1 \mathrm{~K}$ signal when the central beam is completely filled with a $1 \mathrm{~K}$ source. The system is calibrated using cryogenic cold loads and has been checked in the field performing hot/cold load tests and raster scans of the moon and of the Gunn source. The calibrations are consistent to about $20 \%$ absolute accuracy.

\section{OBSERVATIONS AND DATA PROCESSING}

The observations were carried out at Observatorio del Teide in Tenerife (Spain) during 1994 June and July. This observing site is at an altitude of $2400 \mathrm{~m}$ and has been shown to have extremely good atmospheric transparency and stability (Watson et al. 1997). The precipitable water vapor during the campaign was below $1.5 \mathrm{~mm}$ for $10 \%$ of the time. The observed region of the sky was the strip at declination $\delta=40^{\circ}$ where we collected about 550 hours of observations. This declination has been extensively measured from this site at larger angular scales $\left(\sim 5^{\circ}\right)$ and lower frequencies $(10,15$, and $33 \mathrm{GHz}$ ) with reported detections of structures in the CMB by the Tenerife experiments (Hancock et al. 1994).

The experiment was operated only during the night to avoid contamination from solar radiation. Fluctuations in the atmospheric emission are the main source of random noise in our system. The rms of the data collected during several consecutive hours shows that the sky noise of the data binned to $10 \mathrm{~s}$ was of the order of 5.1, 6.8, 11.5, and $13.6 \mathrm{mK}$ for channels $1,2,3$, and 4 , respectively, during typical observing nights. We experienced few nights with excellent observing conditions during which the noise dropped down by a factor $\sim 3.5$ in all channels. Even in these cases the atmospheric noise is larger than the instrumental noise and therefore a major goal in our processing is to assess and subtract this unwanted source of noise. We can reduce the atmospheric noise in channel 1 and 2 by subtracting the extrapolated signal from channel 4 (1.1 mm band) which is the most sensitive to atmospheric gradients. We also checked that extrapolating channel $3(1.3 \mathrm{~mm})$ which produces similar results. In each channel $i$ we have a superposition of astronomical signal and atmospheric signal: $\Delta T_{\mathrm{ANT}, i}=\Delta T_{\mathrm{ANT}, i}^{\mathrm{astro}}+\Delta T_{\mathrm{ANT}, i}^{\mathrm{atm}}$, where all terms are expressed in antenna temperature $\left(T_{\mathrm{ANT}, i}\right)$ and $\Delta T_{A N T, i}^{\mathrm{atm}}=$ $\alpha_{i} \Delta T_{\mathrm{ANT}, 4}^{\mathrm{atm}}$. Since the bulk of the signal in each channel is due to the atmospheric emission and the sky signal is expected to be much smaller, a linear fit of channel $i$ versus channel 4 provides a very good estimation of $\alpha_{i}$. For each channel we have to solve the following equation to obtain the sky signal:

$$
\Delta T_{\mathrm{ANT}, i}=\frac{f_{i}}{c_{i}} \Delta T_{i}^{\text {astro }}+\left(\Delta T_{\mathrm{ANT}, 4}-\frac{f_{4}}{c_{4}} \frac{1}{\rho_{i 4}} \Delta T_{i}^{\text {astro }}\right) \alpha_{i},
$$

where $f_{i}$ and $f_{4}$ are the atmospheric transparencies at channels $i$ and 4 computed using measurements of the water vapor content, pressure, and temperature of the atmosphere (Cernicharo 1985). $\Delta T_{\mathrm{ANT}, i}$ and $\Delta T_{\mathrm{ANT}, 4}$ are the data in channels $i$ and 4 in antenna temperature units; $\Delta T_{i}^{\text {astro }}$ is the astronomical signal in thermodynamic temperature units; $c_{i}$ and $c_{4}$ are the Rayleigh-Jeans to thermodynamic conversion factors: $\Delta T_{\mathrm{ANT}, i}=1 / c_{i} \Delta T_{i}\left(c_{i}=1.29\right.$,
$1.66,3.66,4.82$ for channels $1-4$, respectively); and $\rho_{i 4}$ is the fraction of the astronomical signals seen at channels $i$ and 4: $\rho_{i 4}=$ 1 for CMB signal (i.e., outside the Galactic plane crossing), $\rho_{i 4} \neq$ 1 in the area of the Galactic plane crossing and evaluated according to our predictions for the Galactic foregrounds. Notice that the method requires a prior knowledge of the fraction of the signal expected in the channel $i$ and in channel $4\left(\rho_{i 4}\right)$. In the case of the crossing of the Galactic plane, we use $\rho_{i 4}$ as obtained from our estimations. However, different models in the literature yield essentially the same values for the ratios $\rho_{i 4}$, and threfore we are confident that the recovered Galactic plane is nearly modelindependent.

The atmospheric cleaning procedure was run on the data binned in $10 \mathrm{~s}$ intervals, so that the noise is dominated by atmospheric noise in all channels. After cleaning, the $10 \mathrm{~s}$ binned scans are binned again to 4 minute bin size, so the beam is sampled with at least three points. The typical rms of these cleaned 4 minute binned scans are 0.33 and $0.29 \mathrm{mK}$ for channels 1 and 2, respectively. These scans have an offset drift (always less than $0.6 \mathrm{mK}$ for both channels) which we remove by fitting and subtracting combinations of sinusoidal functions with periods equal or larger than $72^{\circ}$ in R.A., hence much larger than the scales at which our instrument is sensitive. We then proceed with obtaining the final data set by stacking all individual scans where the rms does not exceed 0.5 and 0.55 $\mathrm{mK}$ for channels 1 and 2, respectively. In this way we finally use 179 and $129 \mathrm{hr}$ of data for channels 1 and 2 in order to form the final data set (33\% and $23 \%$ of the total data). The overlap in the data used in channel 1 and channel 2 amounts to $89 \mathrm{hr}$. Figure 1 shows the results in thermodynamic temperatures obtained for channels 1 ( $a$ and $b$ ) and $2(c$ and $d$ ) in the two sections of data away of the Galactic plane $\left(|b| \gtrsim 15^{\circ}\right)$. The data have been binned in increments of $1^{\circ}$ in R.A. The visual appearance of the results of channel 1 is nearly featureless, with all the points lying below the $2 \sigma$ level while channel 2 possibly shows the presence of signal. The mean error-bar in the results of each channel is $\sim 70 \mu \mathrm{K}$ in a $1^{\circ}$ bin in R.A. In Figure $1 e$ and $1 f$ we plot the sum $[(\mathrm{Ch} 1+\mathrm{Ch} 2) / 2]$ and in $1 g$ and $1 h$ the difference $[(\mathrm{Ch} 1-\mathrm{Ch} 2) / 2]$ of both channels.

\section{GALACTIC FOREGROUNDS}

We have analyzed the Galactic contribution including the synchrotron, free-free, and dust emission. The first two processes can be modeled by extrapolating the low-frequency surveys at $408 \mathrm{MHz}$ (Haslam et al. 1982) and $1420 \mathrm{MHz}$ (Reich \& Reich 1986). We use a single power law to model the joint emission from these processes: $T_{\mathrm{ff}-\mathrm{sync}} \propto \nu^{-\beta}$. In the Galactic plane crossing at R.A. $=305^{\circ}$ the free-free emission is expected to dominate over synchroton because of the presence of the source Cyg A and other unresolved $\mathrm{H}$ II regions, and therefore the spectral index is $\beta \sim 2.1$. This was additionally checked by obtaining the $\beta$ values from the $1420 \mathrm{MHz}$ map, which reproduce the Galactic plane crossings at decl. $=+40^{\circ}$ both in the $33 \mathrm{GHz}$ Tenerife beam-switching scans (Davies, Watson, \& Gutiérrez 1996; Gutiérrez 1992) and in the 53 and $90 \mathrm{GHz} C O B E$ DMR maps. We obtain $\beta=$ $2.11 \pm 0.05$, which is the value that we adopted. With this value of $\beta$ the free-free and synchrotron contamination is negligible at our frequencies. This is consistent with Hancock et al. 1996 which found that at $33 \mathrm{GHz}$ and $\delta=40^{\circ}$ this contamination is less than $8 \mu \mathrm{K}$ on scales of about $5^{\circ}$. The dust contribution was estimated by using the $240 \mu \mathrm{m}$ DIRBE map as Galactic template and extrapolated to our frequencies using 

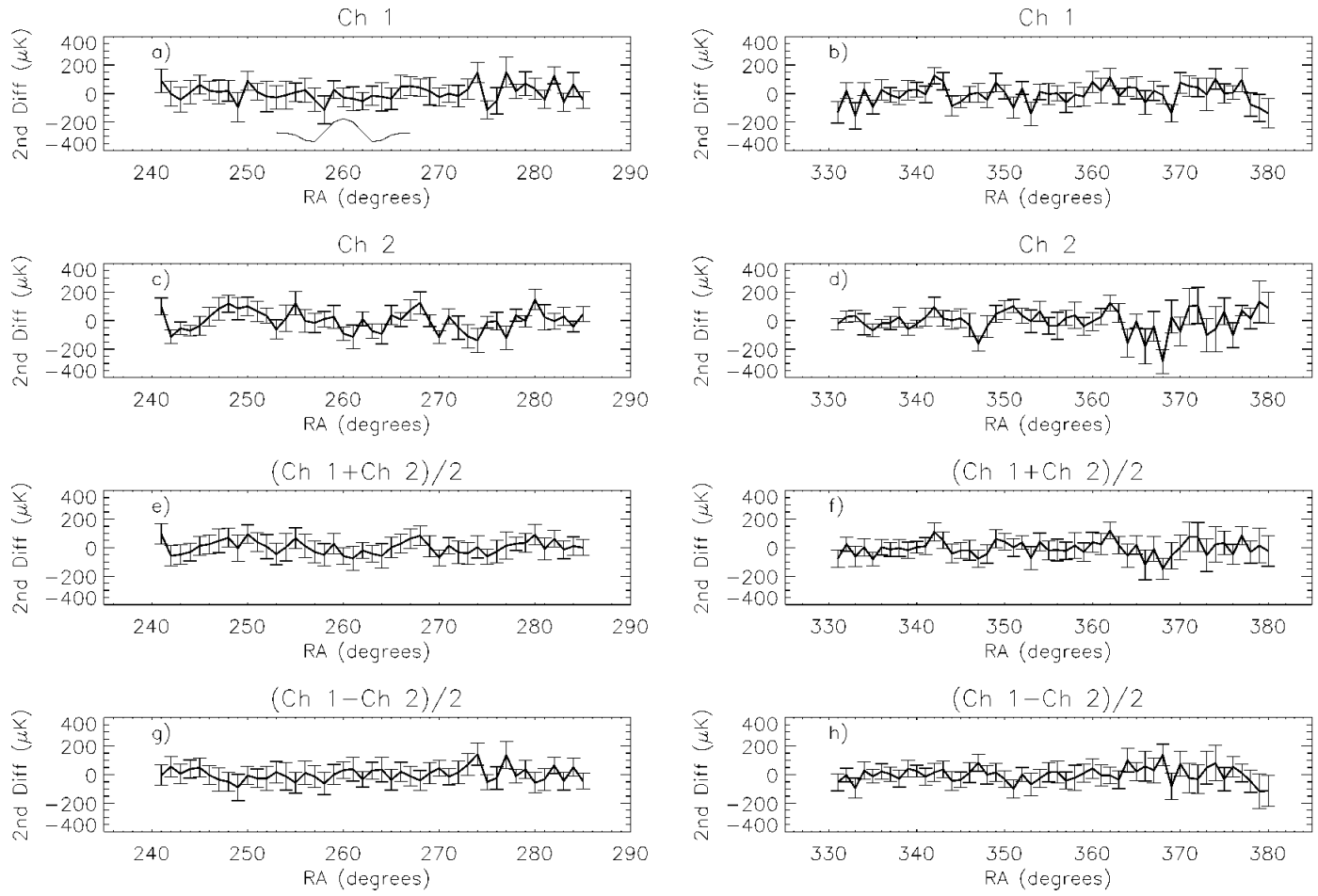

Fig. 1.-Stacked data sets at $3.3 \mathrm{~mm}(a$ and $b)$ and at $2.1 \mathrm{~mm}(c$ and $d)$, the addition ( $e$ and $f)$, and the difference $(g$ and $h)$. In $(a)$, we show the three-beam profile indicating the instrumental response to a point source. Units refer to thermodynamic temperatures in this and following figures.

the model obtained in Boulanger et al. 1996. According to this model the dust emission is well described by a gray body of emissivity $\gamma=2$ (Reach et al. 1995) and with a dust temperature $T_{d}=17.5 \mathrm{~K}: I_{v} \propto \nu^{\gamma} B_{\nu}\left(T_{d}\right)$. In Figure 2 we show the prediction (dashed line) for the Galactic emission and our results for channel 1 (top) and channel 2 (bottom). The general agreement between the predictions and our measurements constitute an important check on the performance of our system and our method of subtracting the atmosphere. Any reasonable combination of $\gamma$ and $T_{d}$ produces a negligible dust contribution outside the area of the main Galactic plane crossing: $\operatorname{rms}_{D}(\mathrm{Ch} 1)=0.5 \mu \mathrm{K}$ and $\mathrm{rms}_{D}(\mathrm{Ch} 2)=1.6 \mu \mathrm{K}$. In this paper we have ignored such contribution. A more detailed analysis will be presented in a forthcoming paper.

\section{STATISTICAL ANALYSIS}

We have analyzed statistically the data of channels 1 and 2 in the ranges $\mathrm{R} . \mathrm{A}_{\cdot_{1}}=241^{\circ}-285^{\circ}$ and $\mathrm{R}$. $\mathrm{A} \cdot{ }_{2}=331^{\circ}-20^{\circ}$ which are at Galactic latitudes $|b| 15^{\circ}$. We computed the correlation function to check for the presence of common structures in both channels. Figure 3 shows the most relevant results obtained. In Figure $3 a$ the cross-correlation between channels 1 and 2 is shown, in Figure $3 b$ we show the autocorrelation of the combined scan $(\mathrm{Ch} 1+\mathrm{Ch} 2) / 2$, and Figure $3 c$ shows the autocorrelation of the difference scan $(\mathrm{Ch} 1-\mathrm{Ch} 2) / 2$. The error-bars in each of the three panels represent the $68 \%$ confidence levels and have been computed using Monte Carlo techniques. Because of the experimental configuration, theexpected correlation in our data, from signals on angular scales at which the instrument is sensitive, will show a characteristic pattern with a positive feature at small angles, followed by a negative bump at angles $\sim 4^{\circ}-10^{\circ}$, and then flat at larger angles. Visual evidence of a signal with this shape exists in both $(a)$ and $(b)$, indicating a possible correlated signal between channels. The solid lines corresponds to the expected correlation for a model estimated using a likelihood analysis (see below). This model has an intrinsic amplitude of $86 \mu \mathrm{K}$ and a coherence angle $\theta_{c}=2{ }^{\circ}$, which corresponds to the amplitude and angle at which the maximum of the likelihood surface is attained for the combined analysis on channel 1 and 2 . In (c)
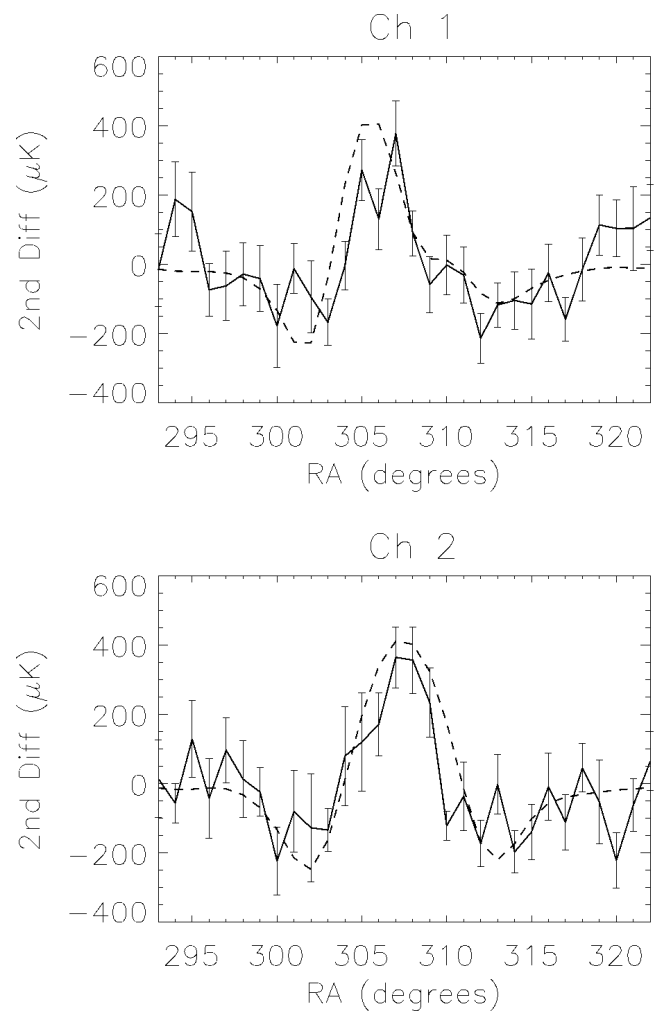

FIG. 2.-Comparison of data at the Galactic plane crossing at $l \sim 80.2^{\circ}$ (solid line) and predictions of foreground emission (dashed line) for channels 1 and 2 (see main text for details). 

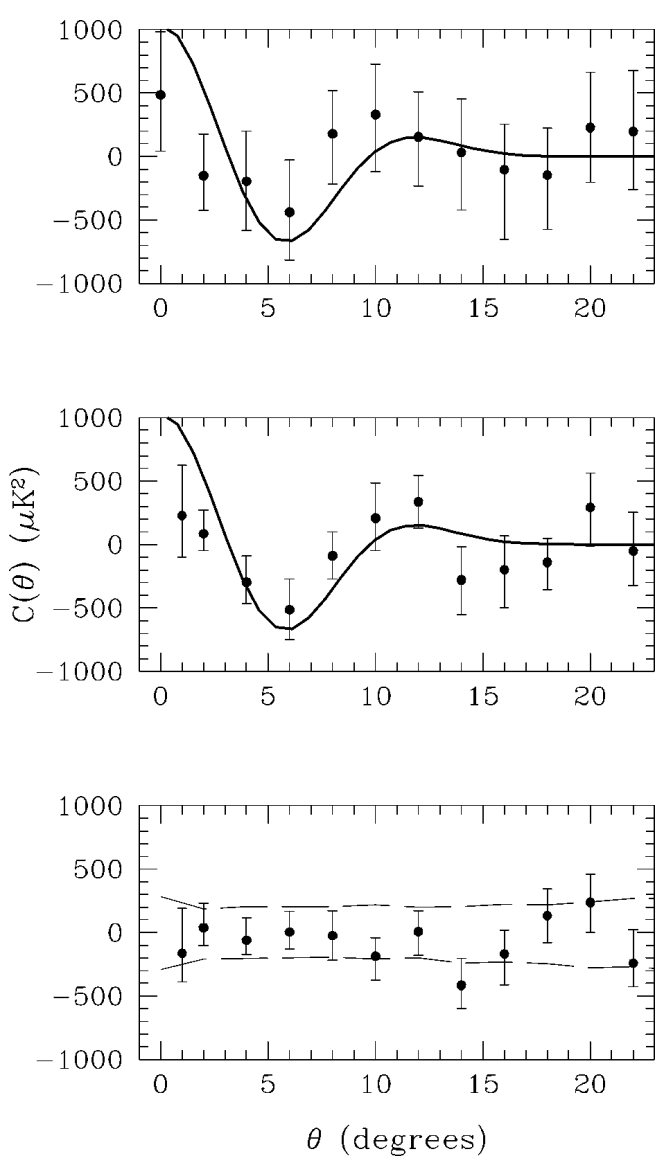

FIG. 3.-Results of the correlation analysis: $(a)$ the cross-correlation of channel 1 with channel $2,(b)$ the autocorrelation of $(\mathrm{Ch} 1+\mathrm{Ch} 2) / 2$, and $(c)$ the autocorrelation of $(\mathrm{Ch} 1-\mathrm{Ch} 2) / 2$. The solid lines in $(a)$ and $(b)$ are the correlation obtained with a likelihood analysis, and the dashed line in $(c)$ is the 95\% confidence limit for uncorrelated noise.

there is no evidence of structure, which is compatible with the expectations in the case of pure uncorrelated noise (dashed bands). Second, a likelihood analysis was used to determine the amplitude and origin of the signals. Our analysis assumed a Gaussian autocorrelation function (GACF) for the CMB signal: $C_{\text {intr }}(\theta)=C_{0} \exp \left(-\theta^{2} / 2 \theta_{c}^{2}\right)$. The GACF models together with its limitations and connections to more realistic scenarios have been widely discussed in the literature (see White \& Scott 1994), and we adopt it to quote preliminary results, leaving physically motivated models for a future analysis. Table 1 presents a summary of the likelihood results for the coherence angle of highest sensitivity $\left(\theta_{c}=1.32\right)$. Upper limits and detections are quoted at confidence limits of $95 \%$
TABLE 1

LiKELIHOOD RESULTS IN $\mu \mathrm{K}^{\mathrm{CMB}^{\mathrm{a}}}$

\begin{tabular}{crrc}
\hline \hline Channel & R.A.1 & R.A.2 & R.A.1 + R.A.2 \\
\hline Ch1 $\ldots \ldots \ldots \ldots \ldots$ & $<92$ & $<115$ & $<72$ \\
Ch2 $\ldots \ldots \ldots \ldots \ldots$ & $94_{-54}^{+63}$ & $109_{-64}^{+71}$ & $101_{-48}^{+50}$ \\
Ch1 \& Ch2 ..... & $72_{-45}^{+50}$ & $79_{-45}^{+51}$ & $76_{-32}^{+43}$ \\
\hline
\end{tabular}

${ }^{\text {a }}$ A $20 \%$ calibration uncertainty has been included.

and $68 \%$, respectively. The analysis of channel 1 does not show evidence of signal in any of the two ranges considered with a limit $C_{0}^{1 / 2}<72 \mu \mathrm{K}$, while for channel 2 we detect signal in both R.A. ranges. We made also a joint likelihood analysis on channel 1 and 2 assuming that both channels have been measuring the same signal. This gives a detection $C_{0}^{1 / 2}=76_{-21}^{+23}$ $\mu \mathrm{K}$. Including the error in the absolute calibration we obtain $C_{0}^{1 / 2}=76_{-32}^{+43} \mu \mathrm{K}$. Even though these results are compatible, we cannot exclude a possible frequency dependence of the detected signals. A two-dimensional (in the plane $C_{0}-\theta_{c}$ ) joint analysis of both channels shows a well defined peak at $C_{0}^{1 / 2}=$ $86 \mu \mathrm{K}, \theta_{c}=2{ }^{\circ}$ : ; the expected correlation in our data for such a signal is plotted as the solid lines in Figures $3 a$ and $3 b$. The likelihood analysis shows the presence of a clear signal in channel 2, while channel 1 is compatible with noise; this indicates a source of non-CMB contaminant in channel 2 . It is unlikely that the data are contaminated significantly by the Galaxy (see previous section). If we convert our results from $\left(C_{0}^{1 / 2}, \theta_{c}\right)$ to band power estimates, we find $[\ell(\ell+1)$ $\left.C_{\ell} / 2 \pi\right]^{1 / 2}=7.7_{-5.1}^{+11.3} \times 10^{-10}$, marginally consistent with the standard CDM model prediction of $1.7_{-0.2}^{+0.3} \times 10^{-10}$ at our $\bar{\ell}=$ 53 (Steinhardt 1994). The excess of signal seen in Ch2 might indicate that some residual atmospheric noise is still contaminating our results. Therefore, we also quote an upper limit of $118 \mu \mathrm{K}$ at a $95 \%$ confidence limit. A more robust atmospheric subtraction technique together with a spectral index analysis of the measured fluctuations has been applied to our data set confirming that part of the signal seen in $\mathrm{Ch} 2$ is of atmospheric origin. We will report these new results in a future paper.

This work has been supported by a University of Delaware Research Foundation (UDRF) grant, by the Bartol Research Institute, and spanish DGICYT project PB 92-0434-c02 at the Instituto de Astrofísica de Canarias. We want to thank L. Page and S. Meyer for considerable help in all the phases of this project. A special thanks for the support of L. Shulman, J. Poirer, R. Hoyland, and the technical staff of the Observatorio del Teide. Finally we would like to thank the staff from the Instituto Nacional de Meteorología at Tenerife who very kindly provided us with the atmospheric data used in this analysis.

\section{REFERENCES}

Bennett, C. L., et al. 1996, ApJ, 464, L1

Boulanger, F., Abergel, A., Bernard, J.-P., Burton, W. B., Désert, F.-X.,

Hartmann, D., Lagache, G., \& Puget, J.-L. 1996, A\&A, 312, 256

Cernicharo, J. 1985, IRAM Int. Rep. 52

Davies, R. D., Watson, R. A., \& Gutiérrez, C. M. 1996, MNRAS, 278, 925

Gundersen, J. O., et al. 1995, ApJ, 443, L57

Gutiérrez, C. M. 1992, Ph.D., Univ. de La Laguna

Hancock, S., et al. 1994, Nature, 367, 333

. 1996, MNRAS, submitted

Haslam, C. G. T., et al. 1982, A\&AS, 47,

Netterfield, C. B., Devlin, M. J., Jarosik, N., Page, L., \& Wollack, E. J. 1996 preprint (astro-ph/9601197)

Netterfield, C. B., Jarosik, N., Page, L., Wilkinson, D., \& Wollack, E. J. 1995, ApJ, 445, L69
Piccirillo, L. 1991, Rev. Sci. Instrum., 62, 1293

Piccirillo, L., \& Calisse, P. 1993, ApJ, 411, 529

Reach, W. T., et al. 1995, ApJ, 451, 188

Reich, P., \& Reich, W. 1986, A\&A, 63, 205

Scott, P. F., Saunders, R., Pooley, G., O'Sullivan, C., Lasenby, A. N., Jones, M., Hobson, M. P., Duffett-Smith, P. J., \& Baker, J. 1996, ApJ, 461, L1

Steinhardt, P. J. 1994, in Proc. CWRU Conf., CMB Anisotropies Two Years After $C O B E$ : Observations, Theory, and the Future, ed. L. Krauss (Singapore: World Scientific), 83

Watson, R. A., et al. 1997, in preparation

White, M., \& Scott, D. 1994, in Proc. CWRU Conf., CMB Anisotropies Two Years After $C O B E$ : Observations, Theory, and the Future, ed. L. Krauss (Singapore: World Scientific), 254 